\title{
EXISTENCE THEOREMS FOR HIGHER ORDER BOUNDARY VALUE PROBLEMS
}

\author{
KEITH SCHRADER AND S. UMAMAHESWARAM
}

\begin{abstract}
In this paper the differential equation $y(n)=f(x, y)$ and associated boundary conditions $y^{(r)}\left(x_{i}\right)=y_{i r}$ for $i=1,2, \cdots, k$ and $r=0,1, \cdots, \lambda(i)-1$ where $\lambda(1)+\lambda(2)+\cdots+\lambda(k)=n$ are considered. Sufficient conditions on $f$ are given to insure the existence of a solution to this $k$ point boundary value problem. In the special cases when $k=2$ or $k=3$ sufficient conditions on $f$ are given to insure both uniqueness and existence of solutions for certain of the boundary value problems.
\end{abstract}

1. Introduction. For $n \geq 2$ we are interested in the $n$th order nonlinear ordinary differential equation

$$
y^{(n)}=f(x, y)
$$

where $f:(a, b) \times R \rightarrow R$ is continuous. The boundary conditions

$$
y^{(r)}\left(x_{i}\right)=y_{i r}, \quad r=0,1, \ldots, \lambda(i)-1, i=1, \ldots, k,
$$

where $\Sigma_{i=1}^{k} \lambda(i)=n, k \geq 2$ and $a<x_{1}<x_{2}<\cdots<x_{k}<b$ will also be considered.

Our purpose in this paper is to obtain sufficient conditions for the existence of a solution to the problem (1.1) and (1.2) on $\left[x_{1}, x_{k}\right]$. The results of this kind which can be found in the literature often fall into one of two cases. In one case the sufficiency conditions require the existence of certain auxiliary functions which satisfy some in equality relation ships with respect to the function $f$, the boundary conditions (1.2) and each other. Thus the problem of solving the differential equation (1.1) with boundary conditions (1.2) for a single function $y$ is replaced by the problem of solving a system of differential and algebraic inequalities for several functions. Results of this kind may be found in [2, Theorem 7.3], [3, Theorems

Received by the editors November 9, 1973.

AMS (MOS) subject classifications (1970). Primary 34B 15; Secondary 34B 10.

Key words. and phrases. Nonlinear, boundary value problems, higher order, multipoint. 
9 and 10], [5, Theorem 3.1] and [6, Corollary 3.1] among other places.

The other type of results commonly found in the literature either assume that $f$ is uniformly bounded on $(a, b) \times R[5$, Lemma 2.3] or assume conditions that imply $|f|$ is bounded by a function of the form $A(x)|y|+B(x)$ (for example by assuming $f$ satisfies a global Lipschitz condition with respect to $y$ on $(a, b) \times R)$ and that the interval $\left[x_{1}, x_{k}\right]$ is sufficiently small. These results are more explicit than the first type of results described above because it is relatively easy, given $f$, to ascertain whether the hypotheses are satisfied. They do, however, severely restrict the growth of $f$ in the $y$ variable in order to assure the existence of a solution to (1.1) and (1.2). When $n=2$, considerably better results are known; for example if $f$ is continuous and nondecreasing in $y$ for each fixed $x$ then all boundary value problems of the form (1.1), (1.2) are solvable [2, Corollary 4.20]. In fact for $n=2$ an even better result is known; namely, if $f$ is continuous and there is an $M>0$ such that $f(x, y) \geq-M$ for $x_{1} \leq x \leq x_{2}$ and $y \geq 0$ and such that $f(x, y) \leq M$ for $x_{1} \leq x \leq x_{2}$ and $y \leq 0$ then (1.1), (1.2) has a solution [6, Corollary 3.2].

Our main existence theorems represent an attempt to generalize the result for $n=2$ just described to the case for arbitrary $n$ and are contained in $\$ 2$. In $\$ 3$ we give existence and uniqueness theorems for some of the boundary value problems (1.1) and (1.2) in the special case when $k=2$ or $k=3$.

2. Multipoint problems. To simplify notation we make the following definition.

Definition 2.1. The symbol $s(j)$ is defined by the equation $s(j)=$ $\sum_{p=1}^{j} \lambda(p)$ where the $\lambda(p)$ are as in equation (1.2).

We now state one of our main existence theorems.

Theorem 2.2. Assume $f$ in (1.1) is continuous on $(a, b) \times R$ and that $f(x, y) \leq M$ for $a<x<b$ and $y \in R$. Let $a<x_{1}<x_{2}<\cdots<x_{k}<b$ and $\lambda(i)$ for $i=1, \cdots, k$ be fixed with $s(k)=n$ and assume $f(x, y) \geq K$ for $x$ in $\left(x_{i}, x_{i+1}\right)$ and $y$ such that $(-1)^{n+s(i)} y \leq 0$. Then (1.1), (1.2) has a solution for all choices of $y_{i r} \in R$.

Proof. Without loss of generality we may assume that $M>0$ and $K<0$. Let $p(x)$ be the unique polynomial of degree $n-1$ satisfying $p^{(r)}\left(x_{i}\right)=y_{i r}$ for $r=0,1, \cdots, \lambda(i)-1$ and $i=1, \cdots, k$. Let $v(x)$ be the solution of the differential equation $v^{(n)}(x)=M$ satisfying $v^{(r)}\left(x_{i}\right)=0$ for $r=0,1, \cdots$, $\lambda(i)-1$ and $i=1, \cdots, k$. Clearly, 


$$
v(x)=M\left(x-x_{1}\right)^{\lambda(1)} \cdots\left(x-x_{k}\right)^{\lambda(k) / n !}
$$

and for $x_{i}<x<x_{i+1}$, Sgn $v(x)=(-1)^{\lambda(i+1)+\cdots+\lambda(k)}=(-1)^{n+s(i)}$ i.e., $(-1)^{n+s(i)} v(x)>0$.

Choose $\phi(x)=p(x)+v(x)$; con sequentily $(-1)^{n+s(i)}\left[\phi(x)^{\prime}-p(x)\right]$ $=(-1)^{n+s(i)} v(x)>0$ on $x_{i}<x<x_{i+1}$. Also $\phi^{(n)}(x)=M \geq f(x, y)$ for all $x$, $y$ with $x_{1} \leq x \leq x_{k}$ and $y \in R$. We will now show that we can choose a function $\psi \in C^{n}\left[x_{1}, x_{k}\right]$ such that $\operatorname{Sgn}[\phi(x)-\psi(x)]=(-1)^{n+s(i)}$ on $x_{i}<x<$ $x_{i+1}$ for $i=1, \cdots, k-1$ and such that $\psi^{(n)}(x) \leq f(x, y)$ for all $(x, y) \in W$ where

$$
\begin{aligned}
W=\bigcup_{i=1}^{k-1}\left\{(x, y): x_{i} \leq x\right. & \leq x_{i+1},(-1)^{n+s(i)} \psi(x) \\
& \left.\leq(-1)^{n+s(i)} y \leq(-1)^{n+s(i)} \phi(x)\right\} .
\end{aligned}
$$

In fact, we shall obtain a function $\psi \in C^{n}\left[x_{1}, x_{k}\right]$ such that $\operatorname{Sgn}[\phi(x)-\psi(x)]$ $=(-1)^{n+s(i)}$ on $x_{i}<x<x_{i+1}$ and $\psi^{(n)}(x) \leq f(x, y)$ for all $(x, y)$ with $x_{i} \leq$ $x \leq x_{i+1}$ and $(-1)^{n+s(i)} y \leq(-1)^{n+s(i)} \phi(x)$ for $i=1, \cdots, k-1$.

By hypotheses $f(x, y) \geq K$ for $x_{i} \leq x \leq x_{i+1}$ and $(-1)^{n+s(i)} y \leq 0$. Let

$$
C_{i}=\operatorname{Max}\left\{(-1)^{n+s(i)} \phi(x): x_{i} \leq x \leq x_{i+1}\right\}
$$

and $C_{i}^{\prime}=\operatorname{Max}\left\{0, C_{i}\right\}$. Then there exists a constant $K_{i} \leq K$ such that $f(x, y)$ $\geq K_{i}$ for $x_{i} \leq x \leq x_{i+1}$ and $(-1)^{n+s(i)} y \leq C_{i}^{\prime}$. Let $K_{0}=\operatorname{Min}\left\{K_{i}: i=1,2\right.$, $\cdots, k-1\}$.

Let $u$ be the solution of the differential equation $u^{(n)}(x)=K_{0}$ satisfying the boundary conditions $u^{(r)}\left(x_{i}\right)=0, r=0,1, \cdots, \lambda(i)-1, i=1,2$, $\cdots, k$. Then

$$
u(x)=\left(K_{0}\left(x-x_{1}\right)^{\lambda(1)} \cdots\left(x-x_{k}\right)^{\lambda(k)}\right) / n !
$$

and for $x_{i}<x<x_{i+1}$,

$$
\text { Sgn } u(x)=(-1)^{\lambda(i+1)+\cdots+\lambda(k)+1}=(-1)^{n+s(2)+1}
$$

i.e., $(-1)^{n+s(i)} u(x)<0$ for $x_{i}<x<x_{i+1}$.

Let $\psi(x)=p(x)+u(x)$, so that we have $\operatorname{Sgn}\lfloor\psi(x)-p(x)\rfloor=(-1)^{n+s(i)+1}$ and $(-1)^{n+s(i)}[\psi(x)-p(x)]<0$ for $x_{i}<x<x_{i+1}$. Thus $(-1)^{n+s(i)} \psi(x) \leq$ $(-1)^{n+s(i)} p(x) \leq(-1)^{n+s(i)} \phi(x)$ for $x_{i} \leq x \leq x_{i+1}$. Now $\psi^{(n)}(x)=u^{(n)}(\dot{x})=$ $K_{0} \leq K_{i} \leq f(x, y)$ for $x_{i} \leq x \leq x_{i+1}$ and $(-1)^{n+s(i)} y \leq C_{i}^{\prime}$.

The functions $\phi$ and $\psi$ together with the differential equation (1.1) and boundary conditions (1.2) satisfy the hypotheses of Theorem 3.1 of [5] so it follows from this theorem that (1.1), (1.2) has a solution $y$ such that $(x, y(x)) \in W$ where $W$ is as defined in equation (2.1). 
Corollary 2.3. Assume $f$ in (1.1) is continuous on $(a, b) \times R$ and that $f(x, y) \leq M$ for $a<x<b$ and $y \in R$. Let $a<x_{1}<x_{2}<\cdots<x_{k}<b$ and $\lambda(i)$ for $i=1,2, \cdots, k$ be fixed with $s(k)=n$. Assume $f\left(x, y_{1}\right) \geq f\left(x, y_{2}\right)$ for $x_{i} \leq x \leq x_{i+1}$ and $(-1)^{n+s(i)}\left(y_{1}-y_{2}\right) \leq 0$. Then (1.1), (1.2) has a solution for all choices of $y_{i r} \in R$.

Proof. If $x_{i} \leq x \leq x_{i+1}$ and $(-1)^{n+s(i)} y \leq 0$ then $f(x, y) \geq f(x, 0)$ so let $K=\operatorname{Min}\left\{f(x, 0): x_{1} \leq x \leq x_{k}\right\}$.

Theorem 2.4. Assume $f$ in (1.1) is continuous on $(a, b) \times R$ and that $f(x, y) \geq M$ for $a<x<b$ and $y \in R$. Let $a<x_{1}<x_{2}<\cdots<x_{k}<b$ and $\lambda(i)$ for $\mathrm{i}=1,2, \cdots, k$ be fixed with $s(k)=n$ and assume $f(x, y) \leq K$ for $x$ in $\left(x_{i}, x_{i+1}\right)$ and $y$ such that $(-1)^{n+s(i)} y \geq 0$. Then (1.1), (1.2) has a solution for all choices of $y_{i r} \in R$.

Proof. The proof of this result is similar to the proof of Theorem 2.2 so it is omitted.

Corollary 2.5. Assume $f$ in (1.1) is continuous on $(a, b) \times R$ and that $f(x, y) \geq M$ for $a<x<b$ and $y \in R$. Let $a<x_{1}<x_{2}<\cdots<x_{k}<b$ and $\lambda(i)$ for $i=1,2, \cdots, k$ be fixed with $s(k)=n$. Assume $f\left(x, y_{1}\right) \leq f\left(x, y_{2}\right)$ for $x_{i} \leq x \leq x_{i+1}$ and $(-1)^{n+s(i)}\left(y_{1}-y_{2}\right) \geq 0$. Then (1.1), (1.2) has a solution for all choices of $y_{i r} \in R$.

Proof. Similar to the proof of Corollary 2.3.

3. Two and three point problems. In this section we consider equations (1.1) and (1.2) when $k=2$ or $k=3$. We need the following lemma due to Kolmogorov [4] in some of the proofs to follow so it is stated here.

Lemma 3.1. Given $M>0,[c, d] \subset R, y \in C^{n}[c, d]$ an arbitrary function with the property that $|y(x)| \leq M$ and $\left|y^{(n)}(x)\right| \leq M$ on $[c, d]$ then there exists a constant $K>0$ depending on $M$ and $d-c$ such that $\left|y^{(r)}(x)\right| \leq K$ on $[c$, d] for $1 \leq r \leq n-1$.

We begin by considering a two point problem with all but one boundary condition specified at $x_{1}$.

Theorem 3.2. Let $f$ in equation (1.1) be continuous on $(a, b) \times R$ and be nondecreasing in $y$ for each fixed value of $x, a<x<b$. Let $k=2, \lambda(1)$ $=n-1$ and $\lambda(2)=1$. Assume that solutions of IVP's (initial value problems) of (1.1) are unique. Let $x_{1}$ be fixed, $a<x_{1}<b$, and $S\left(x_{2}\right)=\left\{y_{2,0}: y_{2,0} \in\right.$ $R$, there exists $y$ satisfying (1.1) and (1.2)\}. Then for each $x_{2}, a<x_{1}<$ 
$x_{2}<b$, either $S\left(x_{2}\right)=\varnothing$ or $S\left(x_{2}\right)=R$. In case $S\left(x_{2}\right)=R$, the solution satisfying (1.1), (1.2) is unique.

Proof. (i) We will first show that if for some $y_{2,0}$ a solution satisfying (1.1) and (1.2) exists, then it is unique.

Assume, if possible, $u$ and $v$ are both solutions of (1.1) and (1.2) and that they are ditsinct. Then since solutions of IVP's are unique we must have $u^{(n-1)}\left(x_{1}\right) \neq v^{(n-1)}\left(x_{1}\right)$. We assume that $u^{(n-1)}\left(x_{1}\right)>v^{(n-1)}\left(x_{1}\right)$. Let $h(x)=u(x)-v(x)$ and let $x_{0}, x_{1}<x_{0} \leq x_{2}$, be the smallest value of $x$ bigger than $x_{1}$ for which $h(x)=0$.

Now $h$ satisfies $h^{(r)}\left(x_{1}\right)=0, r=0,1, \cdots, n-2, h\left(x_{0}\right)=0$ and $h^{(n)}(x)$ $\geq 0$ for $x_{1} \leq x \leq x_{0}$. Using Taylor's theorem with remainder we can write

$$
h(x)=b\left(x_{1}\right)+\sum_{j=1}^{n-1} \frac{h^{(j)}\left(x_{1}\right)}{j !}\left(x-x_{1}\right)^{j}+\frac{h^{(n)}\left(x^{\prime}\right)\left(x-x_{1}\right)^{n}}{n !}
$$

where $x_{1}<x^{\prime}<x \leq x_{0}$ and $x^{\prime}$ depends on $x$. Thus,

$$
h\left(x_{0}\right)=\frac{b^{(n-1)}\left(x_{1}\right)}{(n-1) !}\left(x_{0}-x_{1}\right)^{n-1}+\frac{h^{(n)}\left(x^{0}\right)}{n !}\left(x_{0}-x_{1}\right)^{n}
$$

and hence

$$
h\left(x_{0}\right) \geq \frac{h^{(n-1)}\left(x_{1}\right)}{(n-1) !}\left(x_{0}-x_{1}\right)^{n-1}>0
$$

which is a contradiction. We conclude that $u$ and $v$ must be identical.

(ii) We will now show that for each $x_{2}, a<x_{1}<x_{2}<b, S\left(x_{2}\right)=\varnothing$ or $S\left(x_{2}\right)=R$.

It suffices to show that $S\left(x_{2}\right)$ is both open and closed. To see that $S\left(x_{2}\right)$ is closed, we let $\left\{y_{j}\right\}$ be a sequence of solutions of $(1.1)$ on $\left[x_{1}, x_{2}\right]$ with $y_{j}^{(r)}\left(x_{1}\right)=y_{1 r}, r=0,1, \cdots, n-2$ and $y_{j}\left(x_{2}\right) \rightarrow a$ as $j \rightarrow+\infty$. We will treat the case where $\left\{y_{j}\left(x_{2}\right)\right\}$ is monotone and strictly increasing, the other case being similar. By part (i) of the proof, we have $y_{j+1}(x)>y_{j}(x)$ for $x_{1}<x \leq x_{2}$ Let $h_{j}(x)=y_{j}(x)-y_{1}(x)$ for $j \geq 2$. Then $h_{j}^{(r)}\left(x_{1}\right)=0$ for $r=0$, $1, \cdots, n-2$ and $h_{j}(x)>0$ for $x_{1}<x \leq x_{2}$ and $h_{j}^{(n-1)}\left(x_{1}\right)>0$. Also $h_{j}^{(n)}(x)$ $\geq 0$ for $x_{1} \leq x \leq x_{2}$. Since

$$
h_{j}^{(n-1)}(x)=h_{j}^{(n-1)}\left(x_{1}\right)+\int_{x_{1}}^{x} h_{j}^{(n)}(t) d t,
$$

we also have $h_{j}^{(n-1)}(x)>0$ for $x_{1} \leq x \leq x_{2}$. Then $h_{j}^{(n-2)}(x)=h_{j}^{(n-2)}\left(x_{1}\right)+$ 
$\int_{x}^{x} h_{j}^{(n-1)}(t) d t$ implies $h_{j}^{(n-2)}(x) \geq 0$ for $x_{1} \leq x \leq x_{2}$. Continuing in this way, we see that $h_{j}^{(r)}(x) \geq 0$ for $r=0,1, \cdots, n, x_{1} \leq x \leq x_{2}$ and $j=2,3$, ... From this we see that $h_{j}(x) \leq h_{j}\left(x_{2}\right)$ for $x_{1} \leq x \leq x_{2}$ and hence $\left|h_{j}(x)\right|$ is uniformly bounded for $x_{1} \leq x \leq x_{2}$ and $j \geq 2$. Since $\left\{y_{j}\right\}$ satisfies (1.1) we have $\left|y_{j}^{(n)}(x)\right|$ uniformly bounded for $x_{1} \leq x \leq x_{2}$ and $j \geq 2$.

It now follows from Lemma 3.1 and [1, Theorem 3.2, p. 14] that there is a solution $y$ of $(1.1)$ on $\left[x_{1}, x_{2}\right]$ with $y^{(r)}\left(x_{1}\right)=y_{1 r}, r=0,1, \cdots, n-2$, and $y\left(x_{2}\right)=a$. Hence $a \in S\left(x_{2}\right)$ so $S\left(x_{2}\right)$ is closed.

To see that $S\left(x_{2}\right)$ is open, we assume that $S\left(x_{2}\right)$ is not empty. Then, if $\beta \in S\left(x_{2}\right)$ and $y$ is a solution of (1.1) with $y^{(r)}\left(x_{1}\right)=y_{1 r}, r=0,1, \cdots$, $n-2$, and $y\left(x_{2}\right)=\beta$, it follows that there exists an $\epsilon>0$ such that all solutions $z$ of (1.1) which satisfy $z^{r}\left(x_{1}\right)=y_{1 r}, r=0,1, \cdots, n-2$, and which satisfy $\left|y^{(n-1)}\left(x_{1}\right)-z^{(n-1)}\left(x_{1}\right)\right|<\epsilon$ have a maximal interval of existence $(\omega-, \omega+)$ which contains $\left[x_{1}, x_{2}\right]$. Let $\Delta=\left\{\gamma:\left|y^{(n-1)}\left(x_{1}\right)-\gamma\right|<\epsilon\right\}$ and define $T: \Delta \rightarrow R$ by $T(\gamma)=z\left(x_{2}\right)$ where $z$ is the solution of $(1.1)$ on $\left[x_{1}, x_{2}\right]$ which satisfies $z^{(r)}\left(x_{1}\right)=y_{1 r}, r=0,1, \cdots, n-2$, and $z^{(n-1)}\left(x_{1}\right)=\gamma$. By part (i) of the proof, $T$ is on e-to-one. $T$ is continuous, since solutions of IVP's are unique. It follows that $T$ is a homeomorphism and that $T(\Delta)$ is open. From this it follows that $S\left(x_{2}\right)$ is open.

Now we consider a three point problem with one boundary condition specified at each of the two endpoints and $n-2$ boundary conditions specified at the middle point.

Theorem 3.3. Let $f$ in equation (1.1) be continuous on $(a, b) \times R$ and let $a<x_{1}<x_{2}<x_{3}<b$ with $x_{2}$ fixed. In equation (1.2) assume $k=3, \lambda(1)$ $=\lambda(3)=1$ and $\lambda(2)=n-2$. Assume $f\left(x, y_{1}\right) \geq f\left(x, y_{2}\right)$ for $x_{2} \leq x<b$ and $y_{1} \geq y_{2}$ and assume that $f\left(x, y_{1}\right) \geq f\left(x, y_{2}\right)$ for $a<x \leq x_{2}$ and $(-1)^{n+1}\left(y_{1}-y_{2}\right) \leq 0$. Assume that solutions of IVP's are unique and define $S\left(x_{1}, x_{3}\right)$ for $a<x_{1}<x_{2}<x_{3}<b$ by $S\left(x_{1}, x_{3}\right)=\left\{\left(y_{1,0}, y_{3,0}\right)\right.$ : there exists $y$ satisfying (1.1), (1.2)\}. Then for each pair $\left(x_{1}, x_{3}\right)$ and for each fixed $\left(y_{1,0}, y_{3,0}\right)$ there is at most one solution satisfying (1.1), (1.2). If solutions of IVP's extend to $(a, b)$ then for each pair $\left(x_{1}, x_{3}\right), S\left(x_{1}, x_{3}\right)=R \times R$.

Proof. (i) First we show that if for some pair $\left(y_{1,0}, y_{3,0}\right)$ a solution satisfying (1.1), (1.2) exists, then it is unique.

Assume, if possible, $u$ and $v$ are both solutions of (1.1), (1.2), and that they are distinct. By the uniqueness claimed in Theorem 3.2 we cannot have $u^{(n-2)}\left(x_{2}\right)=v^{(n-2)}\left(x_{2}\right)$. Thus we may assume that $u^{(n-2)}\left(x_{2}\right)>v^{(n-2)}\left(x_{2}\right)$. If $u^{(n-1)}\left(x_{2}\right) \geq v^{(n-1)}\left(x_{2}\right)$ then an argument similar to that in part (i) of 
Theorem 3.2 yields a contradiction. If $u^{(n-1)}\left(x_{2}\right)<v^{(n-1)}\left(x_{2}\right)$, let $h(x)=$ $(-1)^{n}(u(x)-v(x))$ and let $x_{0}, x_{1} \leq x_{0}<x_{2}$ be the largest value of $x$ less than $x_{2}$ for which $h(x)=0$.

Now $h$ satisfies $h^{(r)}\left(x_{2}\right)=0, r=0,1, \cdots, n-3$, Sgn $h^{(n-2)}\left(x_{2}\right)=$ $(-1)^{n}$, Sgn $h^{(n-1)}\left(x_{2}\right)=(-1)^{n+1}, h\left(x_{0}\right)=0, h(x)>0$ for $x_{0}<x<x_{2}$, and $(-1)^{n} \operatorname{Sgn} h^{(n)}(x) \geq 0$ for $x_{0} \leq x \leq x_{2}$. Using Taylor's theorem with remainder we can write

$$
h(x)=b\left(x_{2}\right)+\sum_{j=1}^{n-1} \frac{h^{(j)}\left(x_{2}\right)}{j !}\left(x-x_{2}\right)^{j}+\frac{h^{(n)}\left(x^{\prime}\right)\left(x-x_{2}\right)^{n}}{n !}
$$

where $x_{0} \leq x<x^{\prime}<x_{2}$ and $x^{\prime}$ depends on $x$. Thus,

$$
\begin{aligned}
h\left(x_{0}\right)= & \frac{h^{(n-2)}\left(x_{2}\right)}{(n-2) !}\left(x_{0}-x_{2}\right)^{n-2} \\
& +\frac{b^{(n-1)}\left(x_{2}\right)}{(n-1) !}\left(x_{0}-x_{2}\right)^{n-1}+\frac{h^{(n)}\left(x^{\prime}\right)\left(x_{0}-x_{2}\right)^{n}}{n !}
\end{aligned}
$$

and hence

$$
h\left(x_{0}\right) \geq \frac{b^{(n-2)}\left(x_{2}\right)}{(n-2) !}\left(x_{0}-x_{2}\right)^{n-2}>0
$$

which is a contradiction. We conclude that $u$ and $v$ must be identical.

(ii) We will now show that for each pair $\left(x_{1}, x_{3}\right), a<x_{1}<x_{2}<x_{3}<b$, $S\left(x_{1}, x_{3}\right)=R \times R$.

Since solutions of IVP's extend to $(a, b)$ the solution of (1.1) with initial conditions $y^{(r)}\left(x_{2}\right)=y_{2 r}$ for $r=0,1, \cdots, n-3, y^{(n-2)}\left(x_{2}\right)=0$, $y^{(n-1)}\left(x_{2}\right)=0$ exists on $\left[x_{1}, x_{2}\right]$ so $S\left(x_{1}, x_{3}\right) \neq \varnothing$. Thus it suffices to show that $S\left(x_{1}, x_{3}\right)$ is both open and closed.

To see that $S\left(x_{1}, x_{3}\right)$ is closed we let $\left\{y_{j}\right\}$ be a sequence of solutions of (1.1) on $\left[x_{1}, x_{3}\right]$ with $y_{j}^{(r)}\left(x_{2}\right)=y_{2 r}, r=0,1, \cdots, n-3$, and $y_{j}\left(x_{1}\right) \rightarrow$ $\alpha, y_{j}\left(x_{3}\right) \rightarrow \beta$ as $j \rightarrow+\infty$. Let $h_{j}(x)=y_{j}(x)-y_{1}(x)$. We may now pick a subsequence of $\left\{h_{j}\right\}$, again denoted by $\left\{h_{j}\right\}$, such that either $h_{j}^{(n-2)}\left(x_{2}\right) \geq$ 0 for each $j$ or else $h_{j}^{(n-2)}\left(x_{2}\right)<0$ for each $j$ and such that either $h_{j}^{(n-1)}\left(x_{2}\right) \geq 0$ for each, $j$ or else $h_{j}^{(n-1)}\left(x_{2}\right)<0$ for each $j$. We will treat in detail only the case when $h_{j}^{(n-2)}\left(x_{2}\right) \geq 0$ and $h_{j}^{(n-1)}\left(x_{2}\right) \geq 0$ for each $j$ and the case when $h_{j}^{(n-2)}\left(x_{2}\right) \geq 0$ and $h_{j}^{(n-1)}\left(x_{2}\right)^{j}<0$ for each $j$ since the other two cases are somewhat similar. 
If $h_{j}^{(n-2)}\left(x_{2}\right) \geq 0$ and $h_{j}^{(n-1)}\left(x_{2}\right) \geq 0$ for each $j$ then an argument similar to that in part (i) of the proof of Theorem 3.2 shows that $h_{j}(x) \geq 0$ for $x_{2} \leq x \leq x_{3}$. Also, $h_{j}^{(r)}\left(x_{2}\right)=0$ for $r=0,1, \cdots, n-3$ and $h_{j}^{(n)}(x) \geq 0$ for $x_{2} \leq x \leq x_{3}$. Since

$$
h_{j}^{(n-1)}(x)=h_{j}^{(n-1)}\left(x_{2}\right)+\int_{x_{2}}^{x} h_{j}^{(n)}(t) d t
$$

we also have $h_{j}^{(n-1)}(x) \geq 0$ for $x_{2} \leq x \leq x_{3}$. Then $h_{j}^{(n-2)}(x)=h_{j}^{(n-2)}\left(x_{2}\right)+$ $\int_{x_{2}}^{x} h_{j}^{(n-1)}(t) d t$ implies $h_{j}^{(n-2)}(x) \geq 0$ for $x_{2} \leq x \leq x_{3}$. Continuing in this way we see that $h_{j}^{(r)}(x) \geq 0$ for $r=0,1, \cdots, n, x_{2} \leq x \leq x_{3}$, and $j \geq 2$. From this we see that $h_{j}(x) \leq h_{j}\left(x_{3}\right)$ for $x_{2} \leq x \leq x_{3}$ and hence $\left|h_{j}(x)\right|$ is uniformly bounded for $x_{2} \leq x \leq x_{3}$ and $j \geq 2$. Since $\left\{y_{j}\right\}$ satisfies (1.1) we have $\left|y_{j}^{(n)}(x)\right|$ uniformly bounded for $x_{2} \leq x \leq x_{3}$ and $j \geq 2$. It now follows from Lemma 3.1 and [1, Theorem 3.2, p. 14] that there is a solution $y$ of (1.1) on $\left[x_{1}, x_{3}\right]$ which satisfies $y^{(r)}\left(x_{2}\right)=y_{2 r}$ for $r=0,1, \cdots, n-3, y\left(x_{1}\right)=a$ and $y\left(x_{3}\right)=\beta$. Thus $(\alpha, \beta) \in S\left(x_{1}, x_{3}\right)$.

If $h_{j}^{(n-2)}\left(x_{2}\right) \geq 0$ and $h_{j}^{(n-1)}\left(x_{2}\right)<0$ for each $j$ then let $q_{j}(x)=$ $(-1)^{n} h_{j}(x)$. An argument similar to that in part (i) of this theorem shows that $q_{j}(x) \geq 0$ for $x_{1} \leq x \leq x_{2}$. Also $q_{j}^{(r)}\left(x_{2}\right)=0$ for $r=0,1, \cdots, n-3$, $(-1)^{n} \operatorname{Sgn} q_{j}^{(n-2)}\left(x_{2}\right) \geq 0, \operatorname{Sgn} q_{j}^{(n-1)}\left(x_{2}\right)=(-1)^{n+1}$ and $(-1)^{n} \operatorname{Sgn} q_{j}^{(n)}(x) \geq$ 0 for $x_{1} \leq x \leq x_{2}$. Since

$$
q_{j}^{(n-1)}(x)=q_{j}^{(n-1)}\left(x_{2}\right)+\int_{x_{2}}^{x} q_{j}^{(n)}(t) d t
$$

we have Sgn $q_{j}^{(n-1)}(x)=(-1)^{n+1}$ for $x_{1} \leq x \leq x_{2}$. Then $q_{j}^{(n-2)}(x)=$ $q_{j}^{(n-2)}\left(x_{2}\right)+\int_{x_{2}}^{x} q_{j}^{(n-1)}(t) d t$ implies $(-1)^{n} \operatorname{Sgn} q_{j}^{(n-2)}(x) \geq 0$ for $x_{1} \leq x \leq x_{2}$. Continuing in this way we see that $(-1)^{r} \operatorname{Sgn} q_{j}^{(r)}(x) \geq 0$ for $r=0,1, \cdots, n$, $x_{1} \leq x \leq x_{2}$, and $j \geq 2$. From this we see that $q_{j}(x) \leq q_{j}\left(x_{1}\right)$ for $x_{1} \leq x \leq x_{2}$ and hence $\left|q_{j}(x)\right|$ is uniformly bounded for $x_{1} \leq x \leq x_{2}$ and $j \geq 2$. Since $\left\{y_{j}\right\}$ satisfies (1.1), $\left|y_{j}^{(n)}(x)\right|$ is uniformly bounded for $x_{1} \leq x \leq x_{2}$ and $j \geq 2$. Now by Lemma 3.1 and [1, Theorem 3.2, p. 14] it follows that there is a solution $y$ of $(1.1)$ on $\left[x_{1}, x_{3}\right]$ which satisfies $y^{(r)}\left(x_{2}\right)=y_{2}$, for $r=0,1, \cdots$, $n-3, y\left(x_{1}\right)=\alpha$ and $y\left(x_{3}\right)=\beta$. Thus $(\alpha, \beta) \in S\left(x_{1}, x_{3}\right)$ so $S\left(x_{1}, x_{3}\right)$ is closed.

To see that $S\left(x_{1}, x_{3}\right)$ is open, let $(\alpha, \beta) \in S\left(x_{1}, x_{3}\right)$ and $y$ be a solution of (1.1) with $y^{(r)}\left(x_{2}\right)=y_{2 r}$ for $r=0,1, \cdots, n-3, y\left(x_{1}\right)=\alpha$ and $y\left(x_{2}\right)$ $=\beta$. Define $T: R \times R \rightarrow R \times R$ by $T\left(\gamma_{1}, \gamma_{2}\right)=\left(z\left(x_{1}\right), z\left(x_{3}\right)\right)$ where $z$ is the solution of $(1.1)$ on $\left[x_{1}, x_{3}\right]$ which satisfies $z^{(r)}\left(x_{2}\right)=y_{2 r}, r=0,1, \cdots$, $n-3, z^{(n-2)}\left(x_{2}\right)=\gamma_{1}$ and $z^{(n-1)}\left(x_{2}\right)=\gamma_{2}$. By part (i) of the proof, $T$ is 
one-to-one. $T$ is continuous, since solutions of IVP's are unique. By the Brouwer invariance of domain theorem it follows that $T$ is a homeomorphism and that $S\left(x_{1}, x_{3}\right)=T(R \times R)$ is open.

\section{REF ERENCES}

1. P. Hartman, Ordinary differential equations, Wiley, New York, 1964. MR 30 $\# 1270$.

2. L. Jackson, Subfunctions and second-order ordinary differential inequalities, Advances in Math. 2(1968), 307-363. MR 37 \#5462.

3. G. Klaasen, Differential inequalities and existence theorems for second and third order boundary vlaue problems, J. Differential Equations 10 (1971), 529-537. MR $44 \# 5595$.

4. A. N. Kolmogorov, On inequalities between upper bounds of consecutive derivatives of an arbitrary function on an infinite interval, Učen. Zap. Moskov. Gos. Univ. Matematika 30 (1939), 3-13; English transl., Amer. Math. Soc. Transl. (1) $2^{\circ}$ (1962), 233-243. MR 1, 298.

5. K. Schmitt, Boundary value problems and comparison theorens for ordinary differential equations, SIAM J. Appl. Math. 26 (1974), 670-678.

6. K. Schrader, Boundary-value problems for second-order ordinary differential equations, J. Differential Equations 3 (1967), 403-413. MR 35 \#6893.

7. S. Umamaheswaram, Boundary value problems for higher order differential equations, J. Differential Equations (to appear).

DEPARTMENT OF MATHEMATICS, UNIVERSITY OF MISSOURI, COLUMBIA, MISSOURI 65201 